\title{
Evaluación Morfológica del Músculo Subclavio en una Muestra de Material de Autopsia. Inferencias Clínicas
}

\author{
Morphological Evaluation of the Subclavian Muscle in \\ a Sample of Autopsy Material. Clinical Inferences
}

Edna Rocío Buitrago Quiñonez ${ }^{1,2}$; Iván Darío Quintero Pabón ${ }^{1,2}$ \& Luis Ernesto Ballesteros Acuña ${ }^{1}$

\begin{abstract}
BUITRAGO, Q. E. R.,; QUINTERO, P. I. D. \& BALLESTEROS, A. L. E. Evaluación morfológica del músculo subclavio en una muestra de material de autopsia. Inferencias clínicas. Int. J. Morphol., 39(6):1559-1563, 2021.

RESUMEN: El músculo subclavio (MS) guarda estrecha relación con las estructuras neurovasculares (ENV) en el tercio medio de la región clavicular, situación de suma importancia para la realización de procedimientos invasivos de esta región. Pocos estudios han determinado la distancia desde el MS hacia la vena subclavia (VS), arteria subclavia (AS) y plexo braquial (PB). El propósito de este estudio fue valuar la expresión morfológica del MS y su relación con las ENV en una muestra de especímenes cadavéricos frescos. Estudio descriptivo en el que se realizó disección anatómica sobre la región clavicular de 30 especímenes de cadáveres humanos frescos no reclamados del Instituto Nacional de Medicina Legal y Ciencias Forenses, Colombia. Se realizó la caracterización cualitativa y cuantitativa del MS y se determinó la relación del MS con las ENV en la región clavicular. El MS presentó una longitud de 102,4士10,3

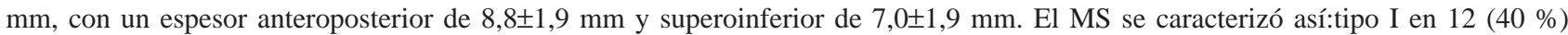
especímenes, tipo II en 18 especímenes, tipo IIa: 10 especímenes. No encontramos tipos III y IV. La VS, AS y PB se relacionaron con el MS en el tercio medial y medio de su longitud a una distancia desde el margen superior del MSde 9,4 $\pm 2,2 \mathrm{~mm}, 11,3 \pm 2,1 \mathrm{~mm}$ y $12,0 \pm 1,5$ mm respectivamente. Los MS se relacionaron con las ENV de la región clavicular en una zona comprendida entre el 36,2 $\pm 3,6 \%$ y $89,4 \pm 4,8 \%$ de su longitud total. Los hallazgos cualitativos y morfométricos de este estudio enriquecen los conceptos anatómicos subclaviculares y son de utilidad para el manejo quirúrgico de fracturas de clavícula.
\end{abstract}

PALABRAS CLAVE: Músculo subclavio; Vena subclavia; Arteria subclavia; Plexo braquial; Clavícula.

\section{INTRODUCCIÓN}

El músculo subclavio (MS) se ubica en la extensión de la cara inferior de la clavícula, se origina mediante una estructura musculotendinosa desde la primera costilla y su unión condrocostal, toma una trayectoria oblicua superior hasta alcanzar su inserción lateral. Piyawinijwong \& Sirisathira (2010) describieron cuatro variantes en la inserción del músculo subclavio. Tipo I, el vientre muscular se inserta en el surco de la cara inferior de la clavícula. Tipo II, la inserción se extiende hasta el ligamento conoide (LC) y la raíz del proceso coracoideo (PC). Tipo III, la inserción se continúa hasta el ligamento superior transverso de la escápula y el Tipo IV, alcanza el vientre inferior del músculo omohioideo. El tipo IV, se conoce también como la variante subclavia posticus reportado en la literatura con una incidencia del 2,3\%-4,8 \% (Martin et al., 2008; Diwan et al., 2014; Grigorita et al., 2018), esta variante se ha relacionado como una causa de síndromes de atrapamiento en la región clavicular. La ubicación del MS y su inserción lateral en la región del hombro le confieren una función de estabilización de la cintura escapular durante el movimiento de abducción, en el cual participa la clavícula realizando movimientos de rotación interna y traslación sobre su eje (Crepaz-Eger et al., 2019).

Se ha reportado la relación de riesgo de la arteria subclavia (AS), vena subclavia (VS) y del plexo braquial (PB) durante los traumas directos y durante los procedimientos quirúrgicos en la región clavicular por su proximidad de estas estructuras neurovasculares (ENV) con el tercio medio de la clavícula (Lo et al., 2010; Rosati et al., 2013; Bain et al., 2013; Jiménez et al., 2017), sin embargo, existe escasa información en relación con la función de protección del MS sobre estas ENV al paso por la región clavicular.

\footnotetext{
${ }^{1}$ Departamento de Ciencias Básicas, Escuela de Medicina,Universidad Industrial de Santander, Bucaramanga, Colombia.

${ }^{2}$ Servicio de Ortopedia y Traumatología, Departamento de Cirugía, Escuela de Medicina,Universidad Industrial de Santander, Bucaramanga, Colombia.
} 
La mayoría de las descripciones en la literatura sobre la relación de las ENV y el MS se limitan a reportes de caso de las variantes en su inserción lateral como hallazgos incidentales durante las disecciones en especímenes formolados (Piyawinijwong \& Sirisathira; Grigorita et al.; Martin et al.; Diwan et al.). Se resalta que escasos estudios previos describan las características cualitativas y cuantitativas de esta estructura muscular sin referenciar cuantitativamente su proximidad con las ENV de la región clavicular, dado lo anterior, el propósito de este estudio fue evaluar la expresión morfológica del MS y su relación con las ENV en una muestra de especímenes cadavéricos frescos.

\section{MATERIAL Y MÉTODO}

Estudio descriptivo transversal que determinó la expresión morfológica del MS en 30 especímenes frescos de cadáveres masculinos no reclamados del Instituto Nacional de Medicina Legal y Ciencias Forenses.

Se realizó un abordaje longitudinal sobre el eje de la clavícula teniendo como referencia la articulación esternoclavicular y acromioclavicular. Se realizó disección de piel, tejido celular subcutáneo y fascia, se protegieron los nervios sensitivos supraclaviculares y se liberó de su inserción en la clavícula a los músculos pectoral mayor, pectoral menor y deltoides. Previa visualización y disección del MS se realizó su caracterización cualitativa y cuantitativa teniendo como referencia el eje longitudinal de la clavícula. Se identificaron los vasos subclavios y el plexo braquial y se determinó su relación morfométrica con relación al margen inferior del MS.

Las evaluaciones morfométricas se realizaron con calibrador digital (Mitutoyo $\left.{ }^{\circledR}\right)$, los resultados fueron analizados en EPI-INFO versión 3.5.1. aplicando las pruebas estadísticas de chi cuadrado (x2) y t de Student aceptando un error alfa hasta del $5 \%$. Esta investigación contó con el aval del Comité de Ética de la Universidad Industrial de Santander y del Instituto Nacional de Medicina Legal y Ciencias Forenses

\section{RESULTADOS}

El MS se originó de la primera costilla y su unión costocondral en todos los especímenes. El vientre muscular presentó una longitud de $102,4 \pm 10,3 \mathrm{~mm}$, con un espesor anteroposterior de $8,8 \pm 1,9 \mathrm{~mm}$ y superoinferior de $7,0 \pm 1,9$ $\mathrm{mm}$ medidos en el segmento medio de su longitud. No hubo diferencias estadísticamente significativas de estos parámetros con relación al lado de presentación ( $\mathrm{p}=0.79$ ). Posteriormente, el MS tomó una trayectoria oblicua lateral y ascendente hasta insertarse en el tercio lateral de la clavícula.

Basados en la clasificación de Piyawinijwong S y Sirisathira N, se observó en el $40 \%$ de la muestra (12 especímenes) el MS tipo I con origen tendomuscular, cuyo tendón ubicado en la margen inferior del musculo se desvanecía progresivamente en dirección medio-lateral; la inserción del vientre muscular se observó en toda la extensión del surco subclavio ubicado en la cara inferior de la clavícula (Fig. 1). El MS tipo II estuvo presente en 18 especímenes (60\%), alcanzando su inserción al ligamento conoide (LC) y al proceso coracoideo (PC) (Fig. 2). En 10 especímenes del tipo II $(33,3 \%)$ se evidenció una variante, a la que se denominó subtipo IIa caracterizada porque la superficie inferior del MS fue cubierta en toda su extensión por una ban-

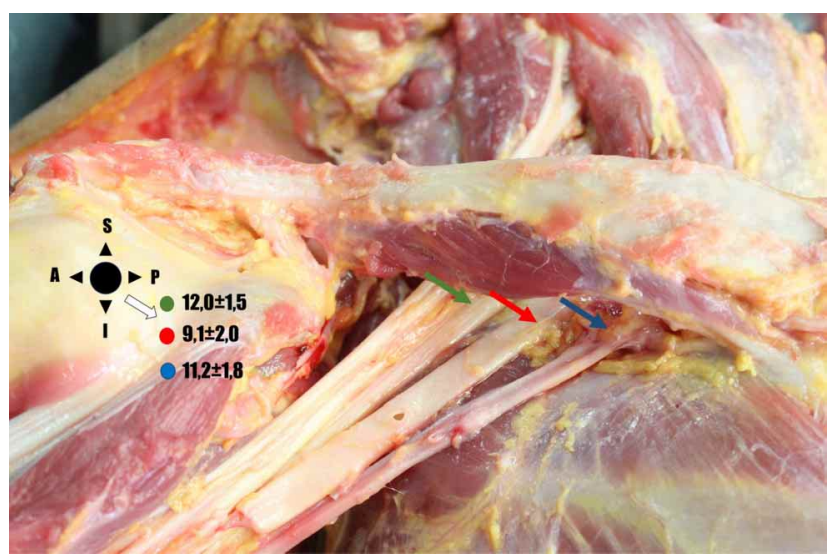

Fig. 1. Región clavicular derecha. (B) Músculo subclavio Tipo I con única inserción a nivel de margen inferior de clavícula. Distancia desde el margen superior del músculo subclavio hasta el punto medio de la VS (Rojo), AS (Azul) y PB (Verde).

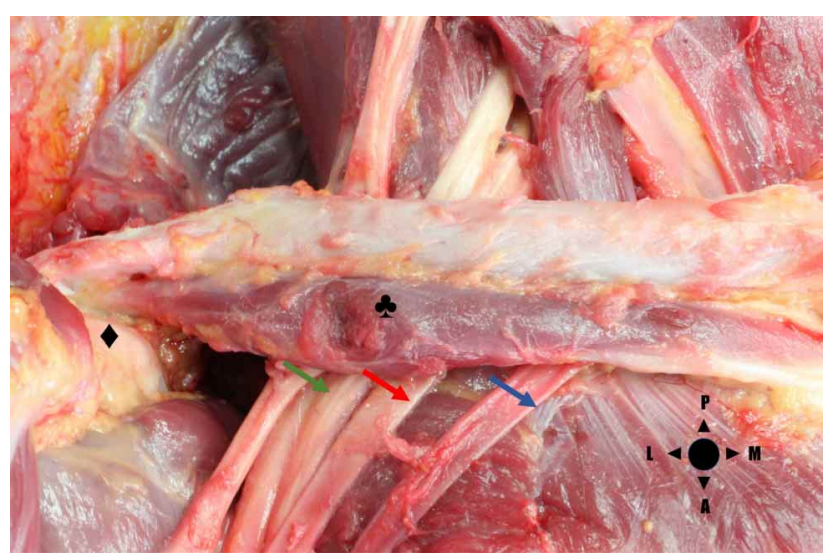

Fig. 2.Región clavicular vista anterior. (ß) musculo subclavio Tipo II. Con inserción a nivel de proceso coracoideo (®) proceso coracoideo. Flechas señalan estructuras neurovasculares azul vena subclavia, roja arteria subclavia, verde plexo braquial. 
da fibro-tendinosa originada desde la primera costilla y se insertó en el LC y PC (Fig. 3). La expansión lateral al LC

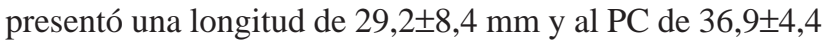
$\mathrm{mm}$, la confluencia de estas fibras generó una configuración triangular cuya base sobre la coracoides presentó una longitud de 22,9 $\pm 4,8 \mathrm{~mm}$ (Fig. 4). En la muestra evaluada no encontramos especímenes con los tipos III y IV.

El MS fue inervado por el Nervio subclavio rama directa del tronco primario superior del PB en todos los especímenes evaluados. La VS, AS y PB, ubicados de medial a lateral, respectivamente, delimitaron una zona comprendida entre el $36,2 \pm 3,6$ y $89,4 \pm 4,8 \%$ de la longitud total del músculo subclavio. La VS, AS, y PB se ubicaron a una distancia desde el margen superior del MS de11,2 $\pm 1,8 \mathrm{~mm}$, $9,1 \pm 2,0 \mathrm{~mm}$ y $12,0 \pm 1,5 \mathrm{~mm}$ respectivamente (Fig. 1). No hubo diferencias estadísticamente significativas de estos parámetros con relación al lado de presentación $(\mathrm{p}=0,21)$.

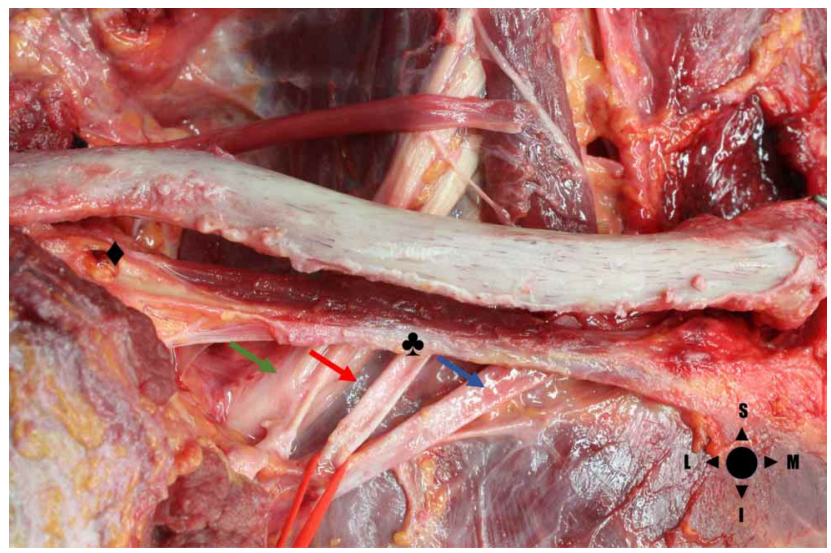

Fig. 3. Región clavicular derecha vista anterior. (B) músculo subclavio Tipo IIa con única banda costocoracoidea. (®) proceso coracoideo. Flechas señalan estructuras neurovasculares azul vena subclavia, roja arteria subclavia, verde plexo braquial.

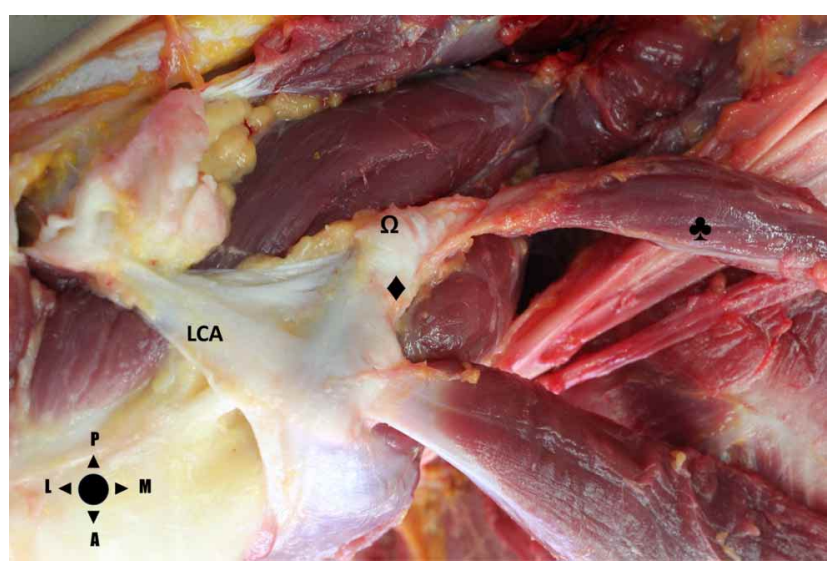

Fig. 4. Región clavicular derecha vista lateral. Inserción lateral triangular del Músculo subclavio (ß) con expansiones al ligamento conoide $(\Omega)$ y proceso coraoideo( $(\circledR)$. Ligamento coracoacromial (LCA).

\section{DISCUSIÓN}

La mayoría de las descripciones en la literatura sobre el MS se limitan a reportes de caso de las variantes en su inserción lateral como hallazgos durante las disecciones en especímenes formolados o durante procedimientos quirúrgicos a nivel del trígono lateral del cuello (Piyawinijwong \& Sirisathira; Grigorita et al.; Martin et al.; Diwan et al.). Piyawinijwong \& Sirisathira realizaron una descripción de las inserciones del MS sobre la cara inferior de la clavícula, LC, PC, ligamento transverso de la escápula y músculo omohioideo, estableciendo de esta manera la clasificación desde los tipos I al IV, siendo el tipo I la más frecuente en el $64 \%$ de los casos. En nuestro estudio predominó el MS tipo II en 18 especímenes (60 $\%$ ), de los cuales 10 presentaron la variante que denominamos tipo IIa correspondiente a una banda fibrosa que cubrió toda la superficie inferior del vientre muscular, característica anatómica descrita previamente como membrana costocoracoidea (Crepaz-Eger et al.). Esta variante del MS podría generar un efecto protector de las estructuras neurovasculares relacionadas en el tercio medio de la clavícula durante los traumas directos y en los procedimientos quirúrgicos sobre el tercio medio de la clavícula ya que los tornillos de las placas de osteosíntesis al quedar incluidos en el vientre muscular o en la banda fibrosa no alcanzan a lesionar a las ENV. En nuestro estudio no se encontraron los tipos III y IV reportados con una incidencia del 2,3-4,8\% (Piyawinijwong \& Sirisathira; Grigoria et al.).

Además de lo señalado en los textos clásicos, los reportes en la literatura sobre la inervación del MS son muy escasos. Jeon et al. (2018) encontraron la inervación del MS fue dada por el nervio subclavio que emergía del tronco superior del plexo braquial en el 95,2 \% de los casos, mientras que en el 4,8 \% esta rama se fusionó con el nervio frénico. En nuestro estudio, la inervación del MS provino del NS como rama directa del tronco primario superior en todos los casos.

EL MS es una estructura en estrecha relación con las ENV de la región clavicular. Robinson et al. (2014) realizaron un estudio anatómico en cadáveres congelados a los que realizaron cortes sagitales en la región clavicular para evaluar la proximidad de la VS, AS y PB con la cortical posterior de la clavícula. En sus hallazgos identificaron que la VS fue la estructura más cercana medialmente a la clavícula a 4,8 mm, mientras que el PB y la AS se encontraron más cercanos a la cortical posterior de la clavícula en el segmento medio y lateral de la misma, a una distancia de $15,2 \mathrm{~mm}$ y $18,6 \mathrm{~mm}$, respectivamente. Sin embargo, Lo et al., en un estudio con especímenes frescos en- 
contraron que la AS y el PB fueron las estructuras más cercanas a la cortical posterior de la clavícula en el tercio medial y medio, con unas dimensiones menores a las reportadas por Robinson et al. $(15,1 \pm 4,7 \mathrm{~mm}$ y $12,76 \pm 4,0$ $\mathrm{mm}$, respectivamente). En nuestros hallazgos, la relación de proximidad de las ENV se midieron con relación al margen superior del MS (VS: $9.1 \pm 2.0 \mathrm{~mm}$, AS: $11.2 \pm 1.8$ $\mathrm{mm}$ y PB: $12.0 \pm 1.5 \mathrm{~mm}$ ), considerando que el MS está estrechamente relacionado a la cortical posteroinferior de la clavícula, se infiere que en nuestro estudio las dimensiones registradas desde el margen superior del MS hacia las ENV son menores a las reportadas fue menor a lo descrito en estudios previos (Galley et al., 2009; Robinson et al.). La variabilidad en las distancias entre la clavícula y el MS con relación a las ENV podría corresponder a las diferentes referencias de medición utilizadas, al fenotipo de los sujetos evaluados, además a la variabilidad del ancho y espesor del vientre muscular del MS y a su distribución sobre la cara inferior de la clavícula, así como al tamaño de las muestras evaluadas en cada estudio. Por las dimensiones de espesor, ancho y longitud señaladas en diferentes estudios, sumados a su particular ubicación topográfica permiten señalar que el MS juega un papel importante de protección en los traumas directos y durante los procedimientos quirúrgicos sobre el tercio medio de la clavícula. La relación del MS con las ENV de la región clavicular en una zona comprendida entre el $36,2 \pm 3,6 \%$ y $89,4 \pm 4,8 \%$ de su longitud total, medida en este estudio, no había sido señalada previamente.

Se han reportado casos de lesiones iatrogénicas intraquirúrgicas o tardías que comprometen o lesionan algunas de las estructuras subclaviculares durante las osteosíntesis de fracturas de la clavícula (Rosati et al.; Bain et al.; Jiménez et al.). Estos resultados cualitativos y morfométricos de este estudio desarrollados en especímenes frescos permiten considerar al MS como referente anatómico durante los procedimientos quirúrgicos sobre el tercio medio de la clavícula a fin de disminuir importantes complicaciones.

\section{AGRADECIMIENTOS}

Los autores agradecen a todos los donantes y sus familias para la realización de esta investigación, cuyos resultados contribuirán a mejorar el conocimiento anatómico y la atención de pacientes. Agradecemos igualmente, al Instituto Nacional de Medicina Legal y Ciencias Forenses por su apoyo en la realización de este estudio en especial a nuestro amigo y compañero Dr. Pedro Luis Forero Porras, víctima fatal por pandemia Covid 19.
BUITRAGO, Q. E. R.,; QUINTERO, P. I. D. \& BALLESTEROS, A. L. E. Morphological evaluation of the subclavian muscle in a sample of autopsy material. Clinical inferences. Int. J. Morphol., 39(6):1559-1563, 2021.

SUMMARY: The subclavian muscle (MS) is closely related to the neurovascular structures (VNS) in the middle third of the clavicular region, and is critical when performing invasive procedures in this region. Few studies have determined the distance from the MS to the subclavian vein (VS), subclavian artery (AS) and brachial plexus (PB). The purpose of this study was to evaluate the morphological expression of MS and its relationship with VNS in a sample of fresh cadaveric specimens. The study involved the anatomical dissection in the clavicular region of 30 specimens of fresh unclaimed human cadavers from the National Institute of Forensic Medicine and Sciences, Colombia. The qualitative and quantitative characterization of the MS was carried out and the relationship of the MS with the VNS in the clavicular region was determined. The MS presented a length of $102.4 \pm 10.3 \mathrm{~mm}$, with an anteroposterior thickness of $8.8 \pm 1.9 \mathrm{~mm}$ and a superoinferior thickness of $7.0 \pm 1.9 \mathrm{~mm}$. The DM was characterized as follows: type I in 12 (40\%) specimens, type II in 18 specimens, type IIa: 10 specimens. We did not find types III and IV. The SV, AS and PB were related to the MS in the medial and middle third of its length at a distance from the upper edge of the MS of $9.4 \pm 2.2 \mathrm{~mm}, 11.3$ $\pm 2.1 \mathrm{~mm}$ and $12.0 \pm 1,5 \mathrm{~mm}$ respectively. The MS were related to the VNS of the clavicular region in an area between $36.2 \pm 3.6$ $\%$ and $89.4 \pm 4.8 \%$ of its total length. The qualitative and morphometric findings of this study enrich the subclavicular anatomical concepts and are useful for the surgical management of clavicle fractures.

KEY WORDS: Subclavian muscle; Subclavian vein; Subclavian artery; Brachial plexus; Clavicle.

\section{REFERENCIAS BIBLIOGRÁFICAS}

Bain, G. I.; Eng, K. \& Zumstein, M. A. Fatal air embolus during internal fixation of the clavicle: a case report. JBJS Case Connect., 3(1):e24, 2013.

Crepaz-Eger, U.; Lambert, S.; Hoermann, R.; Hengg, C.; Knierzinger, D. $\&$ Brenner, E. The anatomy and variation of the coracoid attachment of the subclavius muscle in humans. JSES Open Access, 3(4):251-2, 2019.

Diwan, R. K.; Chopra, J.; Anitarani; Rani, A.; Sehgal, G. \& Srivastava, A. A rare variation of subclavius muscle. J. Anat. Sci., 22(2):22-5, 2014.

Galley, I. J.; Watts, A. J. \& Bain, G. I. The anatomic relationship of the axillary artery and vein to the clavicle: a cadaveric study. J Shoulder Elbow Surg., 18(5):e21-5, 2009.

Grigorita, L.; Vaida, M. A. \& Jianu, A. An aberrant subclavius posticus muscle: a case report. Med. Princ. Pract., 27(6):579-81, 2018.

Jeon, A.; Seo, C. M.; Choi, Y. J.; Lee, J. H. \& Han, S. H. Innervation of subclavius muscle: an anatomical study. Int. J. Morphol., 36(3):81720, 2018.

Jiménez, I.; Marcos-García, A.; Muratore-Moreno, G. \& Medina, J. Neurovascular Complications in Clavicle Fractures Plating: A Simple Procedure to Avoid Them. Tech. Shoulder Elbow Surg., 18(4):129-31, 2017. 
BUITRAGO, Q. E. R.,; QUINTERO, P. I. D. \& BALLESTEROS, A. L. E. Evaluación morfológica del músculo subclavio en una muestra de material de autopsia. Inferencias clínicas. Int. J. Morphol., 39(6):1559-1563, 2021.

Lo, E. Y.; Eastman, Y.; Tseng, S.; Lee, M. A. \& Yoo, B. J. Neurovascular risks of anteroinferior clavicular plating. Orthopedics, 33(1):21, 2010.

Martin, R. M.; Vyas, N. M.; Sedlmayr, J. C. \& Wisco, J. J. Bilateral variation of subclavius muscle resembling subclavius posticus. Surg. Radiol. Anat., 30(2):171-4, 2008

Piyawinijwong, S. \& Sirisathira, N. Supernumerary subclavius muscle in Thais: predisposing cause of thoracic outlet syndrome. J. Med. Assoc. Thai., 93(9):1065-9, 2010.

Robinson, L.; Persico, F.; Lorenz, E. \& Seligson, D. Clavicular caution: an anatomic study of neurovascular structures. Injury, 45(12):1867-9, 2014

Rosati, M.; Andreani, L.; Poggetti, A.; Zampa, V.; Parchi, P. \& Lisanti, M. Progressive brachial plexus palsy after osteosynthesis of an inveterate clavicular fracture. J. Orthop. Case Rep., 3(3):18-21, 2013.
Dirección para correspondencia:

Edna Rocío Buitrago Quiñonez, MD.

Unidad de anatomía

Departamento de Ciencias Básicas

Escuela de Medicina

Universidad Industrial de Santander

Bucaramanga

COLOMBIA

E-mail: e.buitrago.52@gmail.com

Recibido : 22-07-2021

Aceptado: 07-09-2021 